\title{
OPTIMIZED FILTERBANK DESIGN FOR SUBBAND IDENTIFICATION WITH OVERSAMPLING
}

\author{
Damian Marelli and Minyue Fu \\ Department of Electrical and Computer Engineering \\ University of Newcastle, N.S.W. 2308 Australia
}

\begin{abstract}
In this paper, we study the problem of filterbank design for the subband identification method in the oversampled case. We aim to design the filterbanks such that the coupling between adjacent subbands is minimized. This will allow the subband model of the system to be diagonal, thus simplifying the complexity of identification. Solutions are given to two minimization criteria: the worst-input case and whiteinput case.
\end{abstract}

\section{INTRODUCTION}

The linear system identification problem has been extensively studied [1]. Algorithms based on least-squares are commonly employed in practice and their behaviors are well understood. However, the direct use of this algorithm is unsuitable for real-time applications where high order finite impulse response (FIR) models are required (e.g. speech echo cancellation and channel equalization).

These difficulties have motivated a new line of research on system identification that uses subbands $[2,3]$. Loosely speaking, the subband approach divides the input and output signals into a number of subbands using two filter banks. Then for each subband channel, a model is identified. Finally, the subband models are combined to give a full-band model. It is known that the subband approach leads to improvement in computational cost savings, convergence rate and residual error, when the system to be identified has a long impulse response.

If the subband filters are non-ideal, the so called "crossmodels" are needed to modél interference between different subbands (2.5) [2]. In such case, the subband model is said to be coupled. Decoupled subband identification, in the critically sampled case (number of subbands equals the downsampling factor), requires that the filters have very sharp band edges. This drastically increases the filter tap size and therefore the computational cost. In the oversampled case (number of subbands greater than the downsampling factor), the filters are allowed to have a non-zero transition band and therefore smaller tap sizes.
In this work, we consider an oversampled subband identification scheme, with a decoupled subband model. As said before, in order to perfectly identify the fullband system with a decoupled subband model, we need the subband filters to be ideal. In spite of that, we will consider non-ideal FIR filters, and introduce the optimization criteria for the subband filter design, in order to minimize the identification error. This is done for the worst-input case and for the white-input case.

\section{PRELIMINARIES}

\subsection{Frames}

The following are some basic definitions and results about frames. For a more detailed presentation see [4].

Definition 1 Let $\mathcal{H}$ be a separable Hilbert space. A set $\left\{e_{i} \in \mathcal{H}: i \in \mathcal{I}\right\}$ is a frame if there exist constants $A, B>$ 0 such that

$$
A\|x\|^{2} \leq \sum_{i \in \mathcal{I}}\left|\left\langle x, e_{i}\right\rangle\right|^{2} \leq B\|x\|^{2} \quad(\forall x \in \mathcal{H})
$$

The tightest $A$ and $B$ are denoted by $\bar{A}$ and $\bar{B}$, respectively. The operator $T: \mathcal{H} \rightarrow l^{2}(\mathcal{I})$ defined by $(T x)_{i}=$ $\left\langle x, e_{i}\right\rangle \quad(\forall i \in \mathcal{I})(\forall x \in \mathcal{H})$ is called the frame operator:

Remark 2 Denote by $T^{*}$ the adjoint operator of $T$. Then the pseudo-inverse of $T$ is given by $T^{+}=\left(T^{*} T\right)^{-1} T^{*}$, i.e., $T^{+} T$ is the identity operator. It is obvious that $\|T\|=\bar{B}^{1 / 2}$; $\left\|T^{+}\right\|=\bar{A}^{-1 / 2}$

Proposition 3 Given a frame $\left\{e_{i} \in \mathcal{H}: i \in \mathcal{I}\right\}$ and the associated frame operator $T$, there exists a set $\left\{e_{i}^{+} \in \mathcal{H}\right.$ : $i \in \mathcal{I}\}$ such that for any $c \in l^{2}(\mathcal{I})$,

$$
T^{+} c=\sum_{i \in \mathcal{I}} c_{i} e_{i}^{+}
$$

The set $\left\{e_{i}^{+}\right\}$above is called the dual frame of $\left\{e_{i}\right\}$. 


\subsection{Filterbank Approaches}

Consider the filterbank scheme in figure 1 which involves an analysis filterbank and a synthesis filterbank.

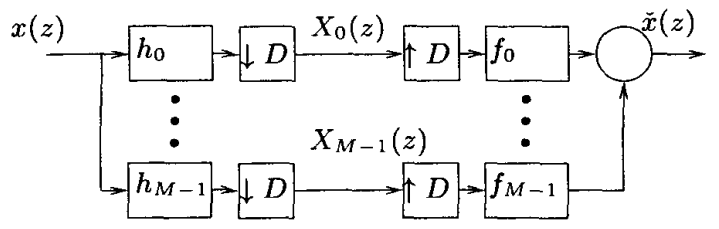

Fig. 1. Filterbank Block Diagram

Filterbanks can be studied and understood using different approaches. In this work we will use the alias approach and the frame approach.

Alias approach: The output $\check{x}(z)$ can be written as

$$
\check{x}(z)=\frac{1}{D} f^{T}(z) H_{A}^{T}(z) X_{A}(z)
$$

where

$$
\begin{aligned}
& X_{A}(z)=\left[x(z) x(W z) \cdots x\left(W^{D-1} z\right)\right]^{T} \\
& f(z)=\left[f_{0}(z) f_{1}(z) \cdots f_{M-1}(z)\right]^{T} \\
& H_{A}(z)=\left[\begin{array}{lll}
h_{0}(z) & \cdots & h_{M-1}(z) \\
\vdots & \ddots & \vdots \\
h_{0}\left(W^{D-1} z\right) & \cdots & h_{M-1}\left(W^{D-1} z\right)
\end{array}\right]
\end{aligned}
$$

and $W=e^{-j \frac{2 \pi}{D}}$. Which means that the filterbank behaves as a linear time-invariant system from the input $X_{A}(z)$ to the output $\check{x}(z)$.

Frame approach: In order for the synthesis filterbank to be perfectly reconstructing, it is required that

$$
\frac{1}{D} f^{T}(z) H_{A}^{T}(z)=[1,0, \ldots, 0] z^{-\tau}
$$

where $\tau$ represents the reconstruction time delay. Define $b(z)=z^{-\tau} f(z)$. Then $b(z)$ is a perfect reconstructing synthesis filterbank with no time delay. Using the frame approach, we define

$$
e_{i j}(t)=h_{i}^{*}(j D-t) \in \mathcal{H},(i, j) \in \mathcal{I}
$$

where $\mathcal{I}=\{(i, j): i=0, \ldots, M-1 ; j \in \mathbb{Z}\}$ and $\mathcal{H}=$ $l^{2}(\mathbb{Z})$. Then $\left\{e_{i j},(i, j) \in \mathcal{I}\right\}$ is a frame. It can be checked that (2.1) can be guaranteed by taking $b(z)$ such that.

$$
b_{i}(t-j D)=e_{i j}^{+}(t),(i, j) \in \mathcal{I}
$$

where $\left\{e_{i j}^{+}\right\}$is the dual frame of $\left\{e_{i j}\right\}$. We will see later that this choice of $b(z)$ not only satisfies (2.1) but also has additional nice properties. The input-output relationship is given by

$$
\check{x}=T_{\tau} T^{+} T x
$$

where $T, T^{+}$are the frame operators for $\left\{e_{i j}\right\}$, and $\left\{e_{i, j}^{+}\right\}$, and $T_{\tau}$ is the time delay operator.

\subsection{Subband Identification}

The subband identification scheme is shown in figure 2 , where $g(z)$ represents a linear time-invariant system with

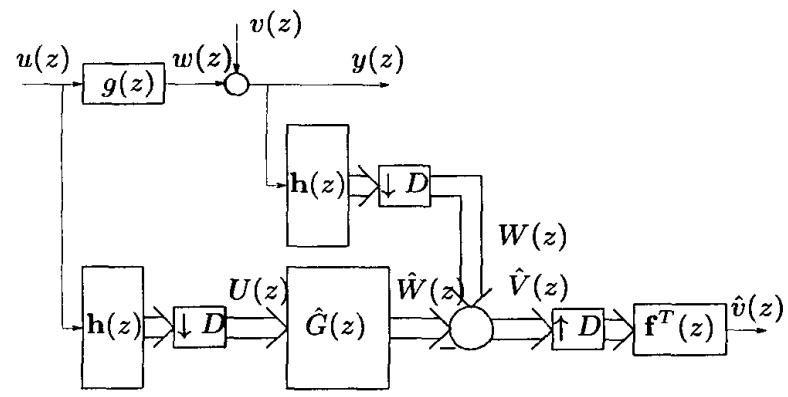

Fig. 2. Subband Identification Block Diagram

input $u(z)$, output $y(z)$ and measurement noise $v(z) ; h(z)=$ $\left[h_{0}(z), \ldots, h_{M-1}(z)\right]^{T}, f(z)=\left[f_{0}(z), \ldots, f_{M-1}(z)\right]^{T}$ are analysis filterbank and synthesis filterbank respectively, and $\hat{G}(z)=\left[\hat{G}_{i j}(z)\right]_{i, j=0}^{M-1}$ represents an equivalent model of $g(z)$ in the subband with down-sampling rate $D$.

Decoupling Condition: To simplify the analysis we assume that $h_{m}(z)=h_{0}\left(V^{m} z\right), m=0, \ldots, M-1$ (i.e. the subband filters come from frequency shifting of a prototype), where $V=e^{-j \frac{2 \pi}{M}}$ and $h_{0}(z)$ is a low-pass filter. In order for the subband model $\hat{G}(z)$ to be diagonal, it is needed that

$$
h_{0}\left(W^{d} z^{1 / D}\right)=0, \quad d=1, \ldots, D-1
$$

The following convention is used throughout this paper: for any $D \in \mathbb{N}$, and $z=r \exp (j \phi), r, \phi \in \mathbb{R}$,

$$
z^{1 / D}=r^{1 / D} \exp \left(j \frac{\bmod (\phi, 2 \pi)-\pi}{D}\right) .
$$

\section{IDENTIFICATION ERROR BOUND}

If (2.5) is satisfied, we only need to identify the diagonal terms of $\hat{G}(z)$. However, (2.5) can only be an approximate in practice due to the fact that non-ideal filters are used. One remedy is also to identify off-diagonal terms of $\hat{G}(z)$. However, this will increase the computational complexity. Alternatively, we can ignore the off-diagonal terms but try to minimize the errors caused by the non-ideal filterbanks. This is what we intend to do in this section. More specifically we consider two cases: the worst-input case and the white-input case. In the sequel we assume that $v(z)=0$.

\subsection{Identification Stage}

Apply the signal $u(z)$ to the input and assume we perfectly identify the input-output relation in each subband. Then, 
using the alias approach,

$\hat{G}_{m m}(z)=\frac{\sum_{d=0}^{D-1} g\left(W^{d} z^{1 / D}\right) \frac{h_{m}\left(W^{d} z^{1 / D}\right)}{h_{m}\left(z^{1 / D}\right)} \frac{u\left(W^{d} z^{1 / D}\right)}{u\left(z^{1 / D}\right)}}{\sum_{d=0}^{D-1} \frac{h_{m}\left(W^{d} z^{1 / D}\right)}{h_{m}\left(z^{1 / D}\right)} \frac{u\left(W^{d} z^{1 / D}\right)}{u\left(z^{1 / D}\right)}}$

for $m=0, \ldots, M-1$.

\subsection{Reconstruction Stage}

Now apply a different input signal $u^{\prime}(z)$. Then,

$$
\begin{aligned}
\hat{V}_{m}^{\prime}(z)= & \frac{1}{D} \sum_{d=0}^{D-1}\left(\hat{G}_{m m}(z)-g\left(W^{d} z^{1 / D}\right)\right) \\
& \left.h_{m}\left(W^{d} z^{1}\right\rangle_{D}\right) u^{\prime}\left(W^{d} z^{1 / D}\right)
\end{aligned}
$$

for $m=0, \ldots, M-1$. We can use the frame approach to write it as

$$
\hat{V}^{\prime}=\tilde{T} u^{\prime}
$$

where $\tilde{T}$ is the map $u^{\prime}(z) \mapsto\left[\hat{V}_{0}^{\prime}(z), \ldots, \hat{V}_{M-1}^{\prime}(z)\right]^{T}$. Now we are ready to state the results for the two cases above.

\subsection{Worst-Input Case Input}

Lemma 4 Consider the mapping in (3.3) and define

$$
\begin{aligned}
& C=\sup _{m, d}\left\{\left\|\left(g\left(W^{d} \Gamma_{m}(z)\right)-g\left(\Gamma_{m}(z)\right)\right) \frac{u\left(W^{d} \Gamma_{m}(z)\right)}{u\left(\Gamma_{m}(z)\right)}\right\|_{\infty}\right\} \\
& F=\sum_{d=1}^{D-1}\left\|h_{0}\left(W^{d} z^{1 / D}\right)\right\|_{\infty} \\
& \text { where } \Gamma_{m}(z)=V^{-m}\left(V^{D m} z\right)^{1 / D} \text {, then } \\
& \qquad\|\tilde{T}\| \leq \frac{C}{\sqrt{D}} F
\end{aligned}
$$

Proof: From (3.2) and taking into account that $h_{m}(z)$ is close to zero outside its support, we have that

$$
\hat{V}_{m}^{\prime}(z) \simeq \frac{1}{D}\left(\hat{G}_{m m}(z)-g\left(\Gamma_{m}(z)\right)\right) h_{m}\left(\Gamma_{m}(z)\right) u^{\prime}\left(\Gamma_{m}(z)\right)
$$

Now, taking into account and that the right hand side of (3.1) does not change if we replace $z^{1 / D}$ by $\Gamma_{m}(z)$, we have that

$$
\hat{G}_{m m}(z)-g\left(\Gamma_{m}(z)\right) \simeq \sum_{d=1}^{D-1}\left(g\left(W^{d} \Gamma_{m}(z)\right)-g\left(\Gamma_{m}(z)\right)\right)
$$

then, combining (3.5) and (3.6)

$$
\begin{aligned}
\hat{V}_{m}^{\prime}(z) \simeq & \left(\frac{1}{D} \sum_{d=1}^{D-1}\left(g\left(W^{d} \Gamma_{m}(z)\right)-g\left(\Gamma_{m}(z)\right)\right)\right. \\
& \left.h_{m}\left(W^{d} \Gamma_{m}(z)\right) \frac{u\left(W^{d} \Gamma_{m}(z)\right)}{u\left(\Gamma_{m}(z)\right)}\right) u^{\prime}\left(\Gamma_{m}(z)\right)
\end{aligned}
$$

Now,

$$
\begin{gathered}
\left\|\hat{V}_{m}^{\prime}(z)\right\|_{2} \leq\left(\frac{C}{D} \sum_{d=1}^{D-1}\left\|h_{m}\left(W^{d} \Gamma_{m}(z)\right)\right\|_{\infty}\right)\left\|u^{\prime}\left(\Gamma_{m}(z)\right)\right\|_{2} \\
=\frac{C}{D} F\left\|u^{\prime}\left(z^{1 / D_{m}}\right)\right\|_{2}
\end{gathered}
$$

since $h_{m}\left(W^{d} \Gamma_{m}(z)\right)=h_{0}\left(W^{d} z^{1 / D}\right)$. Then

$$
\left\|\hat{V}^{\prime}(z)\right\|_{2} \leq \frac{C}{\sqrt{D}} F\left\|u^{\prime}(z)\right\|_{2}
$$

and the result follows immediately from the definition of the norm of a linear operator

We know that if we choose the synthesis filterbank as in (2.3), its associated operator will be the pseudo-inverse of the operator associated with the analysis filterbank. This option has the property that cancels every component of the subband signals that is orthogonal to the range of the analysis filterbank (the subspace of possible subband signals). Clearly, this is the best option for the synthesis filterbank, since it minimizes the energy of $v^{\prime}(z)$ while preserving the perfect reconstruction property. With this choice for the synthesis filterbank, using the frame approach, and in view of (3.3), we can express the reconstruction error as

$$
\hat{v}^{\prime}=T_{\tau} T^{+} \tilde{T} u^{\prime}
$$

Lemma 5 Let $T$ be the frame operator associated with the frame defined by (2.2), and let $\bar{A}>0$ be its tightest lower bound, then

$$
\begin{aligned}
\bar{A}> & \frac{1}{D}\left(\inf _{\omega^{\prime}}\left\{\sum_{m=0}^{M-1}\left|h_{0}\left(\omega-\frac{2 \pi}{M} m\right)\right|^{2}\right\}-\right. \\
& \left.\sum_{d=1}^{D-1}\left(\beta\left(\frac{2 \pi}{D} d\right) \beta\left(-\frac{2 \pi}{D} d\right)\right)\right)^{1 / 2} \equiv \frac{1}{D} E
\end{aligned}
$$

where

$\beta(s)=\sup _{\omega}\left\{\sum_{m=0}^{M-1}\left|h_{0}\left(\omega-\frac{2 \pi}{M} m\right)\right|\left|h_{0}\left(\omega-\frac{2 \pi}{M} m-s\right)\right|\right\}$

Proof: The proof follows the proof of section 3.3 .2 (pp. 67) in $[5]$

Combining lemmas 4 and 5, we have the next result

Theorem 6 The norm of $\hat{v}^{\prime}(z)$ is bounded by

$$
\left\|\hat{v}^{\prime}(z)\right\|_{2} \leq \frac{C}{\sqrt{E}} F\left\|u^{\prime}(z)\right\|_{2}
$$

Proof: Form (3.8) we have that

$$
\left\|\hat{v}^{\prime}\right\|_{2} \leq\left\|T_{\tau}\right\|\left\|T^{+}\right\|\|\tilde{T}\|\left\|u^{\prime}\right\|_{2}
$$

Is easy to see that $\left\|T_{\tau}\right\|=1$. Then the result follows immediately from (3.9) and remark 2 


\subsection{White-Input Case}

If the signal $u^{\prime}(z)$ in the reconstruction stage is a white random process, it can be shown by following similar steps that

$$
\mathcal{E}\left\{\hat{v}^{\prime 2}(t)\right\}=\frac{C}{\sqrt{E}} \hat{F} \mathcal{E}\left\{u^{2}(t)\right\}
$$

where $C$ is in (3.4) and

$$
\hat{F}=\left(\sum_{d=1}^{D-1}\left\|h_{0}\left(W^{d} z^{1 / D_{0}}\right)\right\|_{2}^{2}\right)^{1 / 2}
$$

\section{FILTERBANK DESIGN}

In (3.10) and (3.11), $C$ does not depend on the filter design. Also it can be shown that $E$ does not depend either, provided that the prototype is close to zero in its stop band. Then, the designs should aim to minimize just the term $F$ and $\hat{F}$ respectively.

In summary he filter prototype (see figure 3 ) needs to be:

1. FIR, to be able to use the relation given in [5] to construct the synthesis filterbank.

2. lowpass so as to generate all the $M$ subband filters, from the prototype $h_{0}(z)$, by frequency shifting.

3. approximately a constant value in its passband $\left(-\omega_{1}<\right.$ $\left.\omega<\omega_{1}\right)$.

4. optimized such that $F$ (or $\hat{F}$ ) is as small as possible

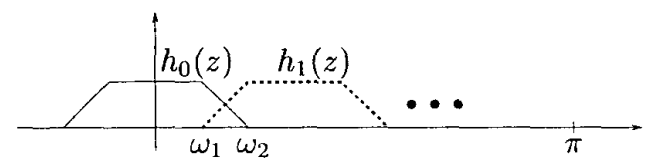

Fig. 3. Filter Prototype

For the worst-input case, the suggested optimization scheme is to start with a Parks-McClellan filter, which gives an equirriple FIR filter. Then, a nonlinear optimization algorithm is used to improve the above filter.

For the white-input case, it is known that the optimal filter that minimizes $\hat{F}$ in (3.12) is an eigenfilter; see [6].

\subsection{Numerical Example}

As an example we consider a subband identification scheme with white inputs, $u(z)$ and $u^{\prime}(z)$. Also, $M=6, D=4$ and $h_{0}(z)$ has a tap size of 20. We compare the norm of $\hat{v}^{\prime}(z)$ for tree filterbank prototypes: the Parks-McClellan filter, the optimized worst-input case filter and the eigenfilter.

Figure 4, shows the frequency response of all tree filters, and figure 5 shows the comparison of the responses in the stopband

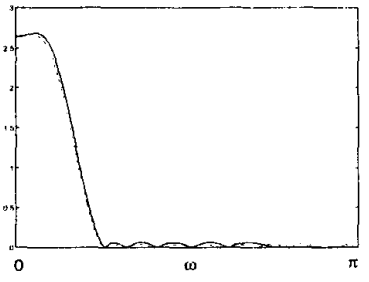

Fig. 4. Optimal prototype

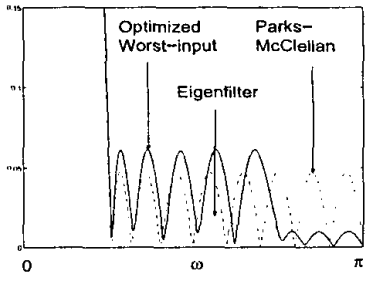

Fig. 5. Stopband detail
The identification error obtained by using the Parks-McClellan filter is $1.04 \times 10^{-3}$, for the optimized worst-input filter is $7.89 \times 10^{-4}$ and for the eigenfilter is $6.55 \times 10^{-4}$. Obviously, the eigenfilter gives the best error because the input $u^{\prime}(z)$ is white. It should be noted that the optimized worstinput case filter may outperform the eigenfilter if $u^{\prime}(z)$ is not white.

\section{CONCLUSION}

In this work we have studied the oversampled subband identification scheme with a decoupled subband model, using FIR filters. We have introduced a bound for the identification error power originated by the use of non-ideal filters. This bound depends on the subband filter prototype. Next, we have used the expression of the bound to find optimal choices to minimize it in both the worst-input case and the white-input case.

\section{REFERENCES}

[1] Lennart Ljung, System Identification: Theory for the User, Prentice Hall, second edition, 1999.

[2] Andre Gilloire and Martin Vetterlli, "Adaptive filtering in subbands with critical sampling: analysis, experiments, and application to acoustic echo cancellation," IEEE Trans. Signal Processing, vol. 40, no. 8, pp. 1862-1875, August 1992.

[3] Youhong Lu and Joel Morris, "Gabor expansion for adaptive echo cancellation," IEEE Signal Processing Magzine, pp. 68-80, March 1999.

[4] Martin Vetterli and Jelena Kovacevic, Wavelets and Subband Coding, Prentice Hall, 1995.

[5] Ingrid Daubechies, "Discrete wavelet transform: frames," in Ten Lectures on Wavelets, chapter 3. Capital City Press, 1992.

[6] P. P. Vaidyanathan, Multirate Systems and Filterbanks, Prentice Hall, 1993. 